
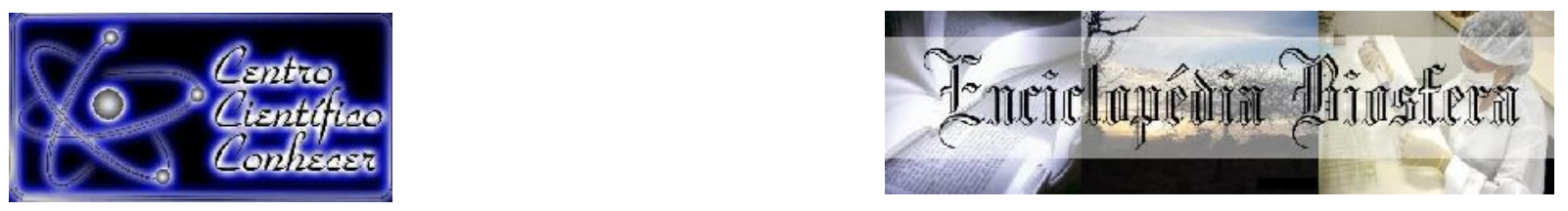

\title{
MORFOANATOMIA FOLIAR DE Plectranthus verticillatus (L.f.) Druce (LAMIACEAE)
}

Elisa Mitsuko Aoyama ${ }^{1}$, Marcos Roberto Furlan ${ }^{2}$, Alexandre Indriunas ${ }^{3}$, Letícia Elias ${ }^{4}$

${ }^{1}$ Professora Doutora da Universidade Federal do Espírito Santo, Centro Universitário Norte do Espírito Santo, São Mateus, Brasil. leticia_dominicini@hotmail.com.

${ }^{2}$ Professor Doutor da Universidade de Taubaté, São Paulo, Brasil.

${ }^{3}$ Doutorando da Universidade Federal do Rio de Janeiro, Museu Nacional, Rio de Janeiro, Brasil.

${ }^{4}$ Graduanda em Ciências Biológicas na Universidade Federal do Espírito Santo, Centro Universitário Norte do Espírito Santo, São Mateus, Brasil.

Recebido em: 15/11/2020 - Aprovado em: 15/12/2020 - Publicado em: 30/12/2020 DOI: 10.18677/EnciBio_2020D48

O gênero Plectranthus fornece importantes espécies de interesse medicinal, entretanto, ainda são poucas as pesquisas sobre seus aspectos botânicos, imprescindíveis para a correta determinação de qual espécie se trata. O objetivo do trabalho foi descrever as características morfoanatômicas foliar de Plectranthus verticilattus para fornecer subsídios para a sua identificação. As folhas foram coletadas em Taubaté-SP. Os parâmetros morfológicos analisados foram: formato, ápice, base, margem e nervação. Amostras foram fixadas em FAA e armazenadas em etanol $70 \%$. Secções a mão livre, com auxílio de lâmina de barbear e isopor foram obtidas no sentido transversal e paradérmico. As lâminas foram preparadas segundo técnicas usuais de anatomia vegetal. Em relação à morfologia, as folhas são orbiculares, com ápice e base obtuso, margem denteada e inteira na base, e nervação com padrão eucamptódromo. Anatomicamente, a folha é hipoestomática com estômatos diacíticos e tricomas glandulares em ambas as faces. Em secção transversal, a nervura central é biconvexa com feixe vascular colateral, o mesofilo dorsiventral com parênquima aquífero, seguido de paliçádico e lacunoso. O pecíolo apresenta tricomas glandulares, além de tectores pluricelulares e colênquima angular abaixo da epiderme. O sistema vascular apresenta dois feixes colaterais maiores e quatro acessórios. As principais características morfoanatômicas foliares observadas no presente trabalho que auxiliam na distinção da espécie são, a forma orbicular do limbo e, no que diz respeito à anatomia, a presença de tricomas tectores somente no pecíolo, seu formato cilíndrico em vista transversal e a presença de parênquima aquífero no mesofilo.

PALAVRAS-CHAVE: pecíolo, planta medicinal, tricoma. 


\title{
LEAF MORPHOANATOMY OF Plectranthus verticillatus (L.f.) Druce (LAMIACEAE)
}

\begin{abstract}
The genus Plectranthus provides important species of medicinal interest, however, there is still little research on its botanical aspects, which are essential for the correct determination of which species it is. The objective of this work was to describe the leaf morphoanatomical characteristics of Plectranthus verticilattus to provide subsidies for its identification. The leaves were collected in Taubaté-SP. The morphological parameters analyzed were: shape, apex, base, margin and nervation. Samples were fixed in FAA and stored in $70 \%$ ethanol. Freehand sections, with the aid of a razor blade and styrofoam were obtained in a transversal and paradermic direction. The slides were prepared according to the usual techniques of plant anatomy. Regarding morphology, the leaves are orbicular, with obtuse base and apex, jagged and whole margin at the base, and nervation with eucamptodromous pattern. Anatomically, the leaf is hypoestomatic with diacitic stomata and glandular trichomes on both sides. In crosssection, the central rib is biconvex with collateral vascular bundle, the dorsiventral mesophyll with an aquiferous parenchyma, followed by palisade and lacunous. The petiole presents glandular trichomes, in addition to pluricellular tectors and angular collenchyma below the epidermis. The vascular system has two larger collateral bundles and four accessories. The main leaf morphoanatomical characteristics observed in the present study that help to distinguish the species are the orbicular shape of the limbus and, with regard to anatomy, the presence of tector trichomes only in the petiole, its cylindrical shape in cross-section and the presence of aquiferous parenchyma in the mesophyll.
\end{abstract}

KEYWORDS: medicinal plant, petiole, trichome.

\section{INTRODUÇÃO}

A caracterização morfoanatômica de uma espécie vegetal é importante para contribuir na sua identificação. Além de ser essencial para evidenciar as diferenças entre uma espécie e outra com a mesma denominação popular, fato comum quando se trata de espécies medicinais (JUDD et al., 2009).

Pertencente à família Lamiaceae, o gênero Plectranthus L'Hér. compreende cerca de 300 espécies distribuídas na Ásia, Austrália e principalmente na África, sendo naturalizada no Brasil (SMITHA et al., 2018). Embora o gênero forneça importantes espécies de interesse medicinal e algumas com vernáculo vulgar em comum, ainda são poucas as pesquisas sobre os aspectos botânicos da maioria destas espécies, como ocorre, por exemplo, com Plectranthus verticillattus (L.f.) Druce (sinonímia Plectranthus nummularius Briq.) (LORENZI; SOUZA, 2008; JUDD et al., 2009).

$P$. verticillatus é usada por algumas comunidades no tratamento de doenças. No estado do Rio de Janeiro, é denominada popularmente por dólar e comercializada como medicinal no comércio informal (LEITÃO et al., 2014). Em Manaus, recebe o nome de trevo-roxo, e a infusão ou chá de suas folhas é utilizado na medicina tradicional para dores de ouvido (BARBOSA et al., 2019). 
É uma herbácea reptante, perene, com folhagem ornamental, originária da Austrália e llhas do Pacífico. A planta pode atingir de 15 a $20 \mathrm{~cm}$ de altura, e as suas inflorescências são terminais, eretas e curtas, com flores pequenas e brancas. Multiplica-se por separação da ramagem prostrada já enraizada em qualquer época do ano, sendo tolerante a baixas temperaturas (LORENZI; SOUZA, 2008).

Em pesquisa sobre etnofarmacologia em Castela Mancha, Espanha, Rivera et al. (2019) levantaram que $P$. verticillatus é encontrada nos jardins domésticos de aldeias e utilizada na medicina tradicional da região. Com relação às pesquisas sobre a comprovação científica de suas atividades farmacológicas, são encontrados poucos estudos. Avaliando extratos de cinco espécies do gênero Plectranthus, Rijo et al. (2012) verificaram que os extratos acetônicos de $P$. madagascarensis, $P$. verticillatus e $P$. hadiensis demonstraram ser ativos exclusivamente em bactérias Gram-positivas. Fouche et al. (2006) em seus estudos descreveram a espécie com potencial para atividade anticâncer.

Devido à importância da espécie, o objetivo do trabalho foi descrever as características morfoanatômicas foliar de Plectranthus verticilattus para fornecer subsídios para a sua identificação.

\section{MATERIAL E MÉTODOS}

De cinco espécimes obtidos em maio de 2020, em Taubaté-SP (23.013S, 45.560W) foram coletadas dez folhas adultas, completamente expandidas e sadias. Os parâmetros morfológicos foliares analisados foram: formato, ápice, base, margem, superfície, filotaxia e nervação segundo terminologia de Hickey (1973) e Ellis et al. (2009), com auxílio de microscópio estereoscópio.

Para os estudos anatômicos, amostras da porção mediana do limbo e pecíolo foram fixadas em FAA 50 (formaldeído: ácido acético: álcool etílico 50\%, 2:1:18, v/v), de acordo com Johansen (1940), mantidas por 48 horas e posteriormente transferidas para etanol $50 \%$. Foram realizadas secções transversais do terço médio foliar e peciolar com auxílio de lâmina de barbear e isopor, além de secções paradérmicas de ambas as faces foliares para estudo das estruturas superficiais.

Posteriormente as secções foram clarificadas com solução de hipoclorito de sódio a 25\%, coradas com azul de alcian 0,5\% e safranina 1\% (LUQUE et al., 1996), e montadas em lâminas semipermanentes com gelatina glicerinada. As lâminas foram analisadas ao microscópio óptico e as imagens obtidas em fotomicroscópio Motic BA 210, com projeção de escalas micrométricas.

\section{RESULTADOS E DISCUSSÃO}

Em relação à morfologia, as folhas são orbiculares, com ápice e base obtuso, margem denteada e inteira na base, nervação com padrão eucamptódromo, coriáceas (FIGURA 1) e filotaxia oposta cruzada. 
FIGURA 1 - Folhas de Plectranthus verticillatus (L. f.) Druce. A. Face adaxial. B. Face abaxial.

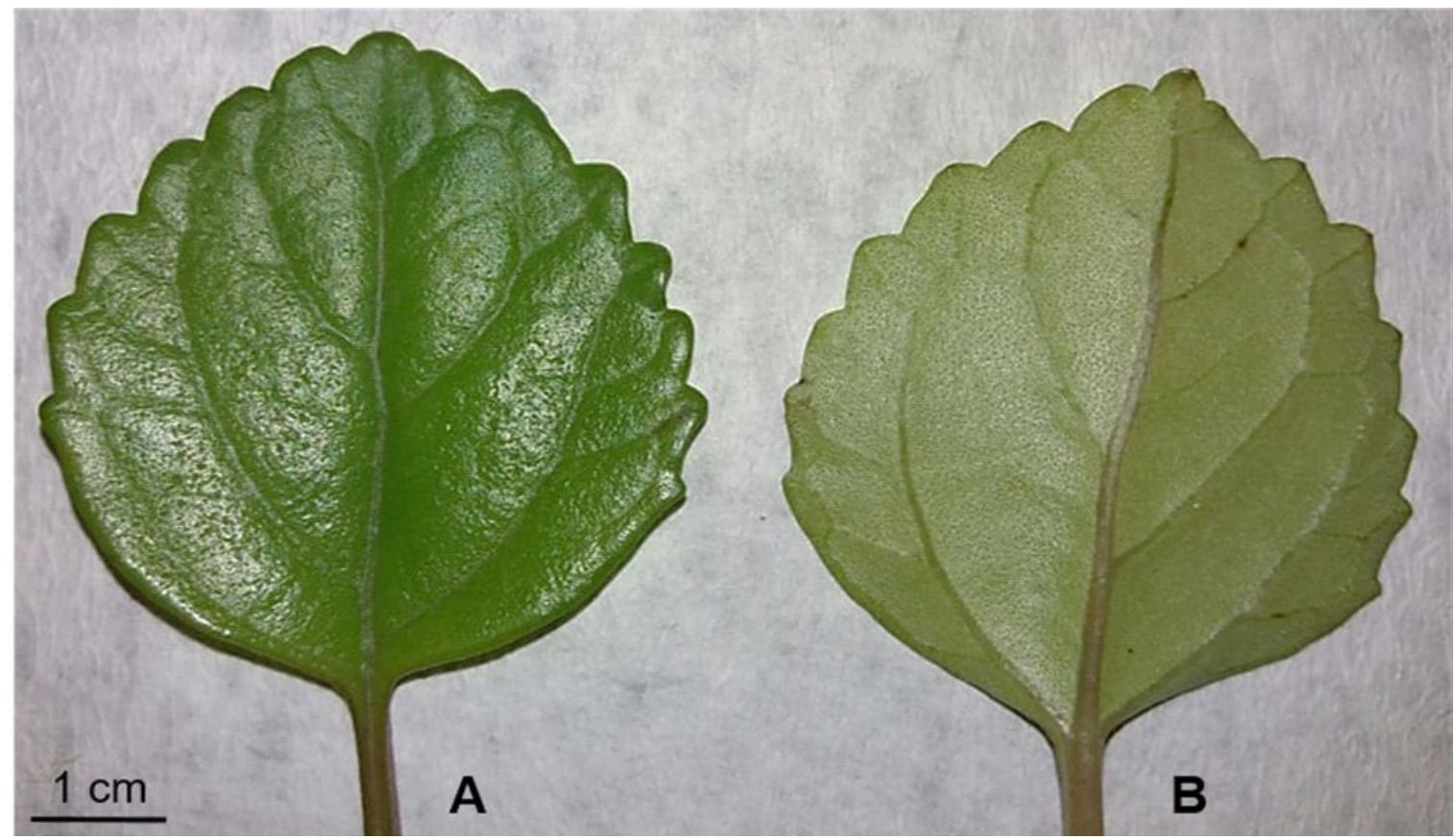

FONTE: autores (2020)

As espécies do gênero Plectranthus apresentam considerável variação morfológica das folhas (SMITHA et al., 2018). Khalik (2016), em seu trabalho com sete espécies do gênero para a Arábia Saudita, observou quatro espécies com limbo de formato subesférico e três ovóide, enquanto Smitha et al. (2018), em seu estudo com $P$. bishopianus o compara com uma espécie próxima, $P$. deccanicus, tendo a primeira limbo ovado e a segunda, cordado. Esses dados demonstram uma relativa variação desse carácter. Dentre as espécies medicinais do gênero empregadas no Brasil, $P$. verticillatus não possui a evidente pilosidade como as demais.

Quanto aos caracteres anatômicos, em vista frontal a epiderme da face adaxial apresenta células poligonais com paredes celulares retas (FIGURA 2A) e na face abaxial, as células possuem as paredes celulares sinuosas (FIGURA 2B), além de tricomas glandulares em ambas as superfícies foliares. As folhas de $P$. verticillatus são hipoestomáticas, com estômatos do tipo diacíticos (FIGURA 2B), diferindo de $P$. barbatus que apresenta estômatos anomocíticos (DUARTE; LOPES, 2007). 
FIGURA 2 - Vista frontal das folhas de Plectranthus verticillatus (L. f.) Druce. A. Face adaxial, tricoma glandular (seta). B. Face abaxial, setas pretas apontam os estômatos e tricomas glandulares (setas vermelhas).
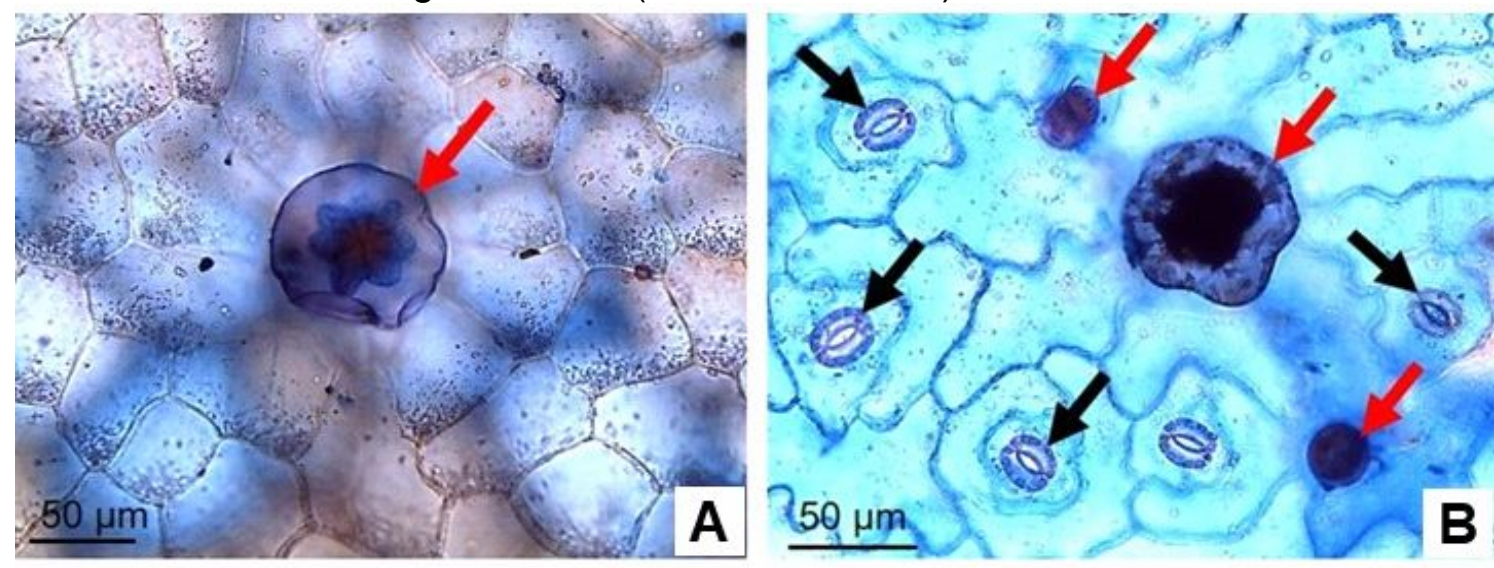

FONTE: autores (2020)

Quanto à posição dos estômatos, o gênero apresenta diversidade, onde Duarte e Lopes (2007) ao compararem a espécie alvo do estudo e $P$. neochilus, com dados de $P$. barbatus, assinalam que ambas são anfiestomáticas. Ramborger et al. (2020), por sua vez, apontam que $P$. neochilus é hipoestomática. No que diz respeito à forma, o gênero apresenta estômatos amonocíticos para $P$. ornatus e P. amboinicus (MAURO et al., 2008) e diacítico, como encontrado na espécie em estudo, e para $P$. neochilus (DUARTE; LOPES, 2007), P. vettiveroides (VELVIZHI et al., 2020) e P. barbatus (SCAVONE, 1965 citado por DUARTE; LOPES, 2007).

Os tricomas são um importante caracter taxonômico para a família Lamiaceae (GUL et al., 2019) assim como para o gênero Plectranthus (KHALIK, 2016), sendo importante para a distinção de espécies (MAURO et al., 2008). Estes possuem ampla variação (KHALIK, 2016). Tricomas tectores são comuns em espécies do gênero como apresentado nos trabalhos de Duarte e Lopes (2007), Mauro et al. (2008), Khalik (2016), Khalik e Karakish (2016), Gul et al. (2019), Ramborger et al. (2020) e Velvizhi et al. (2020), muito embora não tenha sido observado na espécie em estudo, sendo a ausência bastante incomum.

Os tricomas glandulares observados em vista frontal apresentam dois tamanhos relativos, sendo o maior encontrado em ambas as faces (FIGURAS 2A e 2B) e os menores somente na abaxial (FIGURA 2B). Em vista transversal identificam-se dois tipos denominados como: tricomas glandulares subsésseis (FIGURA 3D), os quais possuem célula basal diminuta em relação à porção apical glandular, sendo esta primeira posicionada no mesmo nível das demais células epidérmicas. Os tricomas glandulares peltados (FIGURAS 3E e 3G) apresentam uma porção basal comparativamente mais proeminente a qual ultrapassa as demais células epidérmicas.

Duarte e Lopes (2007), assim como replicado por Ramborger et al. (2020), denominaram o tipo de tricoma glandular subséssil, como peltado enquanto, Mauro et al. (2008) utilizam a mesma terminologia aqui empregada. Tricomas glandulares com porção basal proeminente a qual ultrapassa as demais células epidérmicas são ENCICLOPÉDIA BIOSFERA, Centro Científico Conhecer - Jandaia-GO, v. 17 n.34; p. 626 2020 
encontrados em diversas espécies do gênero, no presente estudo, foi observado que a célula basal possui um aspecto quadrangular de proporção muito próxima à porção apical. Esta característica não foi observada nas espécies da literatura consultada, já citada. Segundo Huchelmann et al. (2017), esses tricomas são especializados na síntese, armazenamento e secreção de metabólitos secundários como terpenóides e flavonóides.

Cabe ressaltar que a alta variedade e tipologias de tricomas são de grande importância não somente para a taxonomia ou em análise em materiais frescos, mas também para a identificação de drogas vegetais, ou seja, material seco e muitas vezes pulverizado, como apontado por Aoyama e Indriunas (2015) para espécies medicinais de Justicia L. (Acanthaceae). Como assinalado por Ramborger et al. (2020), diversas espécies de Plectranthus são amplamente empregadas para fins medicinais, sendo precípua a busca de uma padronização da terminologia e tipologia para tricomas do gênero, bem como das demais Lamiaceae de interesse medicinal.

Em secção transversal, a nervura central é plano-convexa (FIGURA 3A) com parênquima aquífero abaixo da epiderme na face adaxial e parênquima fundamental ao redor do feixe vascular colateral central (FIGURA 3B). O mesofilo é dorsiventral com parênquima aquífero, seguido de 1 a 2 camadas de parênquima paliçádico e de 2 a 3 camadas de lacunoso (FIGURA 3C). O tipo de mesofilo é comum na literatura pesquisada, porém não foram encontradas referências de presença de parênquima aquífero em outros estudos sobre anatomia foliar de Plectranthus.

O pecíolo tem forma cilíndrica (FIGURA 3F) e apresenta tricomas glandulares subsséseis (FIGURA 3G), além de tectores pluricelulares e colênquima angular abaixo da epiderme (FIGURA 3H). O sistema vascular apresenta dois feixes colaterais maiores e quatro acessórios (FIGURAS 3F e 3I). A forma cilindra do pecíolo não foi observada nas espécies analisadas por outros autores (DUARTE; LOPES, 2007; MAURO et al., 2008; KHALIK, 2016; KHALIK; KARAKISH, 2016; VELVIZHI et al., 2020), o que mostra a importância desta característica com distintiva para outras espécies do gênero. Estudos das características da anatomia de pecíolos em Lamiaceae são relevantes para taxonomia como apontado por Rashid e Parnell (2017) em estudo para espécies de Premna L., por Shahri et al. (2016) para Nepeta L., por Noor et al. (2018) Vitex Tour. ex L. e de Jehanzeb et al. (2020), para a tribo Menthae, subfamília Nepetoideae do Paquistão. 
FIGURA 3 - Secções transversais de folhas de Plectranthus verticilattus (L. f.) Druce. A. Nervura central. B. Detalhe do feixe vascular colateral da nervura central. C. Limbo com mesofilo dorsiventral. D. Tricoma glandular subséssil (seta). E. Tricoma glandular peltado (seta). F. Pecíolo. G. Tricoma glandular peltado. H. Colênquima angular. I. Detalhe do feixe vascular colateral do pecíolo.
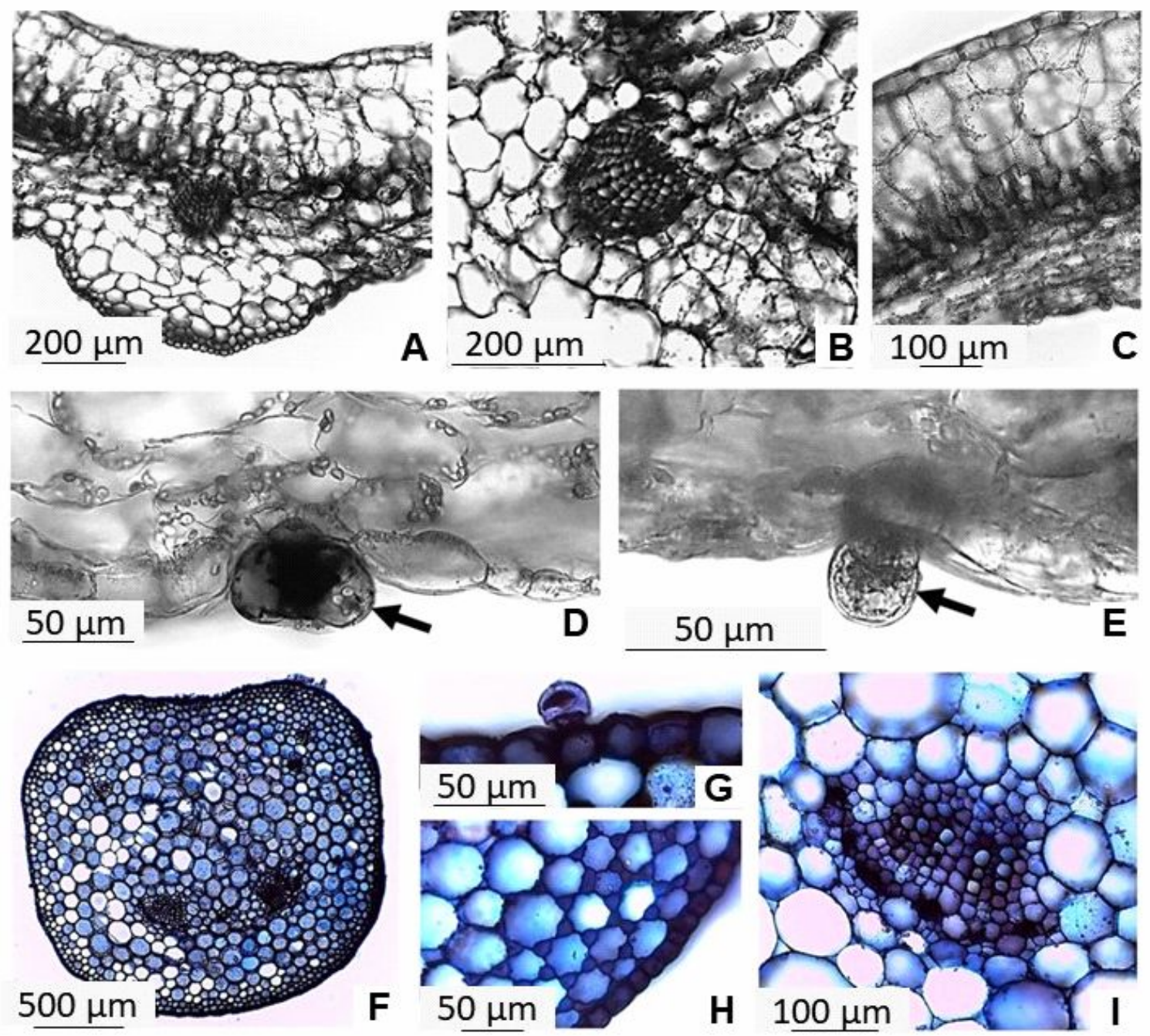

FONTE: autores (2020)

\section{CONCLUSÃO}

As principais características morfoanatômicas foliares observadas no presente trabalho, que contribuem para a distinção de Plectranthus verticillatus de outras espécies do gênero utilizadas no Brasil, são a forma orbicular do limbo e, no que diz respeito à anatomia, a presença de tricomas tectores somente no pecíolo, seu formato cilíndrico em vista transversal e a presença de parênquima aquífero no mesofilo. Deste modo, a forma do limbo auxilia o reconhecimento da espécie a olho nu e os caracteres anatômicos, quando o material se encontra fragmentado. 


\section{REFERÊNCIAS}

AOYAMA, E. M.; INDRIUNAS, A. Use of anatomy for the identification of vegetal drugs from two species of Justicia (Acanthaceae) marketed as "anador". Scientia Plena, v. 11, n. $7, \quad$ p. $1-7, \quad 2015.2$ Disponível em: <https://www.scientiaplena.org.br/sp/article/view/071002/1322>.

BARBOSA, C. S.; SCUDELLER, V. V.; FERREIRA, S. A. N.; BONATTO, E. C. S.; PINTO, E. O. S. Plantas medicinais cultivadas em quintais no Bairro de São Raimundo, da cidade de Manaus, AM. Revista Terceira Margem Amazônia, v. 4, n. 12, p. 122141, 2019.2 Disponível em: <http://www.revistaterceiramargem.com/index.php/terceiramargem/article/view/267/198 $>$.

DUARTE, M. R.; LOPES, J. F. Stem and leaf anatomy of Plectranthus neochilus Schltr., Lamiaceae. Revista Brasileira de Farmacognosia, v. 17, n. 4, p. 549-556, 2007. Disponível em: <https://www.scielo.br/scielo.php?script=sci_arttext\&pid=S0102695X2007000400013>. doi: 10.1590/S0102-695X2007000400013

ELLIS, B.; DALY, D. C.; HICKEY, L. J.; JOHNSON, K. R.; MITCHELL, J. D.; et al. Manual of leaf architecture. New York: New York Botanical Garden Press, 2009. 190 p.

FOUCHE, G.; KHOROMBI, E.; KOLESNIKOVA, N.; MAHARAJ, V. J.; NTHAMBELENI, R.; et al. Investigation of South African plants for anticancer properties. Pharmacologyonline, v. 3, p. 494-500, 2006. Disponível em: < https://pharmacologyonline.silae.it/files/archives/2006/vol3/048.Fouche.pdf>.

GUL, S.; AHMAD, M.; ZAFAR, M.; BAHADUR, S.; CELEP, F.; et al. Taxonomic significance of foliar epidermal morphology in Lamiaceae from Pakistan. Microscopy research and technique, v. 82, n. 9, p. 1507-1528, 2019. Disponível em: <https://onlinelibrary. wiley.com/doi/epdf/10.1002/jemt.23316>. doi: 10.1002/jemt.23316

HICKEY, L. J. Classification of the architecture of dicotyledonous leaves. American Journal of Botany, v. $60, \quad$ n. $1, \quad$ p. 17-33, 1973 . Disponível em: <http://www.u.arizona.edu/ bblonder/leaves/The_secrets_of_leaves/Making_skeletons files/American\%20Journal\%20of\%20Botany\%201973\%20Hickey\%20Classification\%20 of\%20the\%20architecture\%20of.pdf>. doi: 10.2307/2441319

HUCHELMANN, A.; BOUTRY, M.; HACHEZ, C. Plant Glandular Trichomes: Natural Cell Factories of High Biotechnological Interest. Plant Physiology, v. 175, n. 1, p. 6-22, 2017. Disponível em: <http://www.plantphysiol.org/content/plantphysiol/175/1/6.full.pdf>. doi: $10.1104 /$ pp.17.00727

JEHANZEB, S.; ZAFAR, M.; AHMAD, M.; SULTANA, S.; ZAMAN.; et al. Comparative petioler anatomy of tribe Mentheae subfamily Nepetoideae, Lamiaceae from Pakistan. 
Feddes Repertorium, v. 131, n. 3, p. 163-174, 2020. Disponível em: <https://onlinelibrary.wiley.com/doi/abs/10.1002/fedr.202000009>. doi: 10.1002/fedr.202000009

JOHANSEN, D. A. Plant Microtechnique. McGraw- Hill: New York, 1940. 523 p.

JUDD, W. S.; CAMPBELL, C. S.; KELLOGG, E. A.; STEVENS, P. F. Sistemática Vegetal: um enfoque filogenético. 3. ed. Porto Alegre: Artmed, 2009. 632 p.

KHALIK, K. N. A. A systematic revision of the genus Plectranthus L. (Lamiaceae) in Saudi Arabia based on morphological, Palynological, and micromorphological characters of Trichomes. American Journal of Plant Sciences, v. 7, n. 10, p. 14291444, 2016. Disponível em: < https://www.scirp.org/journal/paperinformation.aspx?paperid=68853>. $\quad$ doi: 10.4236/ajps.2016.710137

KHALIK, K. N. A.; KARAKISH, E. A. Comparative anatomy of stems and leaves of Plectranthus L. (Lamiaceae) in Saudi Arabia and systematic implications. Microscopy Research and Technique, v. 79, n. 7, p. 583-594, 2016. Disponível em: < https://onlinelibrary.wiley.com/doi/abs/10.1002/jemt.22671 >. doi: 10.1002/jemt.22671

LEITÃO, F.; LEITÃO, S. G.; FONSECA-KRUEL, V. S.; SILVA, I. M.; MARTINS, K. Medicinal plants traded in the open-air markets in the State of Rio de Janeiro, Brazil: an overview on their botanical diversity and toxicological potential. Revista Brasileira de Farmacognosia, v. 24, n. 2, p. 225-247, 2014. Disponível em: <https://www.scielo.br/scielo.php?script=sci_arttext\&pid=S0102-695X2014000200225>. doi: 10.1016/j.bjp.2014.04.005

LORENZI H.; SOUZA H. M. Plantas ornamentais no Brasil: arbustivas, herbáceas e trepadeiras. 4. ed. Nova Odessa: Instituto Plantarum, 2008. 1088 p.

LUQUE, R.; SOUZA, C. H.; KRAUS, E. J. Métodos de coloração de Roeser (1972) modificado e Kropp (1972) visando a substituição do azul de Astra pelo azul de alcião 8GS ou 8GX. Acta Botanica Brasilica, v. 10, n. 2, p. 199-212, 1996. Disponível em: <http://dx.doi.org/10.1590/S0102-33061996000200001>. doi: 10.1590/S010233061996000200001

MAURO, C., SILVA, C. de P., MISSIMA, J., OHNUKI, T., RINALDI, R. B., et al. Estudo anatômico comparado de órgãos vegetativos de boldo miúdo, Plectranthus ornatus Codd. e malvariço, Plectranthus amboinicus (Lour.) Spreng. - Lamiaceae. Revista Brasileira de Farmacognosia, v. 18, n. 4, p. 608-613, 2008. Disponível em: <https://www.scielo.br/scielo.php?script=sci_arttext\&pid=S0102-695X2008000400019>. doi: 10.1590/S0102-695X2008000400019 
NOOR, N. N. M.; MOHAMED, F.; DAUD, N.; ALIMON, H.; RAHMAN, M. A.; et al. Comparative anatomical study and morphological observation of three medicinal Vitex species (Lamiaceae). Malayan Nature Journal, v. 70, n. 3, p. 285-296, 2018. Disponível

em:

<https://www.researchgate.net/publication/328781048_Comparative_anatomical_and_st udy_and_morphological_observation_of_three_medicinal_Vitex_species_Lamiaceae\#ful ITextFileContent>.

RAMBORGER, B. P.; PAZ, M. E. G.; DENARDIN, E. L. G.; SOARES, J. J.; ROEHRS, R. A review of anatomical, physiological, biological characteristics and uses of Plectranthus neochilus. Ciência e Natura, v. 42, p. e12, 2020. Disponível em: <https://periodicos.ufsm.br/cienciaenatura/article/view/40157/html>. doi: $10.5902 / 2179460 \times 40157$

RASHID, H.; PARNELL, J. A. N. Petiolar anatomical characters and its taxonomic significance in some species of Premna L. (Lamiaceae). Pleione, v. 11, n. 2, p. 405-419, $2017 . \quad$ Disponível em: <https://www.researchgate.net/publication/322575591_Petiolar_Anatomical_Characters and_its_Taxonomic_Significance_in_some_Species_of_Premna_L_Lamiaceae>. doi: 10.26679/Pleione.11.2.2017.405-419

RIJO, P.; BATISTA, M.; MATOS, M. ROCHA, E.; JESUS, S.; et al. Screening of antioxidant and antimicrobial activities on Plectranthus spp. extracts. Biomedical Biopharmaceutical Research, v. 9, n. 2, p. 225-235, 2012. Disponível em: <http://recil.grupolusofona.pt/jspui/bitstream/10437/3689/1/Article11_9n2.pdf>.

RIVERA, D.; VERDE, A.; FAJARDO, J.; OBÓN, C.; CONSUEGA, V.; et al. Ethnopharmacology in the Upper Guadiana River area (Castile-La Mancha, Spain). Journal of Ethnopharmacology, v. 241, n. 15, 2019. Disponível em: <https://www.sciencedirect.com/science/article/pii/S0378874119302661?via\%3Dihu>. doi: 10.1016/j.jep.2019.111968

SHAHRI, S. M. M.; JAFARI, A.; MAHMOODZADEH, H. Comparative Anatomical Studies on Petioles of Nepeta L. Species (Lamiaceae) in NE Iran. Advanced Studies in Biology, v. 8, n. 3, p. 119-126, 2016. Disponível em: <http://www.mhikari.com/asb/asb2016/asb1-4-2016/p/jafariASB1-4-2016-2.pdf>. $\quad$ doi: 10.12988/asb.2016.6728

SMITHA, K.; PATON, A.; SUNOJKUMAR, P. Re-establishment of Plectranthus bishopianus (Lamiaceae) based on morphological and micromorphological data. Plant Systematics and Evolution, v. 304, n. 7, p. 807-816, 2018. Disponível em: <https://link.springer.com/article/10.1007/s00606-018-1511-6>. doi: 10.1007/s00606018-1511-6 
VELVIZHI, D.; NARTUNAI, G.; SUSIKUMAR, S.; ILAVARASAN, R. Morpho-anatomical, Phytochemical and TLC/HPTLC Studies on Whole Plant of Plectranthus vettiveroides (Jacob) NP Singh \& BD Sharma. Pharmacognosy Journal, v. 12, n. 1, p. 58-65, 2020. Disponível em: <http://phcogj.com/sites/default/files/PJ-12-1-279_0.pdf>. doi: 10.5530/pj.2020.12.10 